\title{
The optimized schemes of optical labels about DB and PPM over POLMUX-CSRZ-DQPSK payload in $100 \mathrm{~Gb} / \mathrm{s}$ OLS network
}

\author{
Ying $\mathrm{Li}^{1,}$, Ming $\mathrm{Xu}^{2}$, b , Tiansheng Zhang, Yan Li and Jianhua Ji \\ ${ }^{1}$ School of ShenZhen University, ShenZhen 518060, China; \\ ${ }^{2}$ School of ShenZhen University, ShenZhen 518060, China. \\ a526521165@qq.com, bxum@szu.edu.cn
}

\begin{abstract}
Keywords: optical label switching (OLS), Differential quadrature phase shift keying (DQPSK), duobinary (DB), pulse position modulation (PPM), high bit-rate.
\end{abstract}

\begin{abstract}
Two orthogonal modulation optical label switching (OLS) schemes, which are based on payload of polarization multiplexing-carrier suppressed return to zero-differential quadrature phase shift keying (POLMUX-CSRZ-DQPSK) with label identifications of duobinary (DB) label and pulse position modulation(PPM) label, are proposed in high speed OLS network. The receiver performance of two optical labels and the optimized extinction ratio (ER) are compared with amplitude shift keying (ASK) label by simulation in long transmission distance. The results show DB label has more advantages in fast optical label switching system, and PPM label can improve receiver sensitivity of payload significantly. Two labels have excellent performance in high bit-rate transmission system.
\end{abstract}

\section{Introduction}

Optical labelling using orthogonal modulation formats has been an emerging topic already in the early 1990's, work in Leonid Kazovsky's group at Standford University on the STARNET network [1][2][3], and was revived in the early to mid-2000's, European project STOLAS (Switching Technologies for Optically Labelled Signals), followed by a number of groups worldwide. However, previous studies were based on low bit-rate networks, and $100 \mathrm{~Gb} / \mathrm{s}$ backbone network is applied to commercial network now [4], researches on this high bit-rate transmission network is necessary. In addition, compared with labels which have been proposed, such as amplitude shift keying (ASK) [5], frequency shift keying (FSK) [6], binary phase shift keying (BPSK) [7], and polarization shift keying (PolSK) [8], the duobinary (DB) modulation is known as its high spectral efficiency and easy to detect with direct detection [9][10]. The pulse position modulation (PPM) is sensitive to pulse position and its intensity is constant, so that PPM can solve the extinction ratio (ER) conflict of intensity modulation and phase modulation [11]. Based on the above situation, two optimized orthogonal modulation optical label switching (OLS) schemes are proposed in this paper, in which DB and PPM used as optical labels combined with $100 \mathrm{~Gb} / \mathrm{s}$ polarization multiplexing-carrier suppressed return to zero-differential quadrature phase shift keying (POLMUX-CSRZ-DQPSK) payload.

The remainder of this paper is organized as follows. Simulation of two proposed schemes is shown in section 2. Simulation results and the conclusions are described in Section 3 and section 4, respectively.

\section{Simulation of two proposed OLS schemes}

We round simulation with a commercial software package by an OptiWaves System 7.0. The system architecture is shown in Figure 1. DB label or PPM label is modulated on payload by Mach-Zehnder modulation (MZM), with use of Erbium-doped Optical Fiber Amplifier (EDFA), transmission over single mode fiber (SMF) and dispersion compensation fiber (DCF), and finally label and payload are detected separately. The rate of labels are $2.5 \mathrm{~Gb} / \mathrm{s}$, and payload is $100 \mathrm{~Gb} / \mathrm{s}$. Transmission distance of POLMUX-DQPSK-CSRZ/DB system and 
POLMUX-CSRZ-DQPSK/PPM system are $1560 \mathrm{~km}$ and $3000 \mathrm{~km}$ with designing as balanced dispersion management, respectively. Each loop consists of two DCF, two SMF and four EDFA. Parameters of two systems are list in table1.

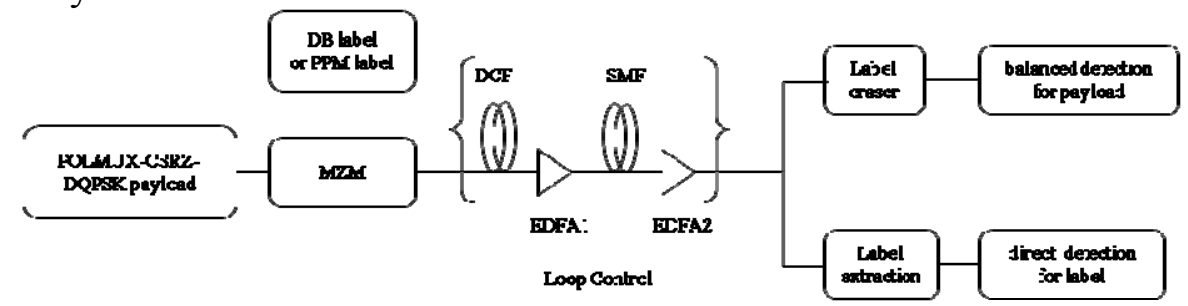

Fig. 1 Schematic of POLMUX-CSRZ-DQPSK/DB and POLMUX-CSRZ-DQPSK/PPM system

Table 1 Parameters of two systems

\begin{tabular}{cc}
\hline parameter & value \\
\hline ER of MZM & $2 \mathrm{~dB}$ for dB label \\
Length of DCF & $0.6 \mathrm{~dB}$ for PPM label \\
& $10 \mathrm{~km}$ \\
Dispersion coefficient of DCF & $-85 \mathrm{ps} / \mathrm{ns} / \mathrm{km}$ \\
Dispersion slope of DCF & $-0.3 \mathrm{ps} / \mathrm{nm} 2 / \mathrm{km}$ \\
Attenuation coefficient of DCF & $0.5 \mathrm{~dB} / \mathrm{km}$ \\
Length of SMF & $50 \mathrm{~km}$ \\
Dispersion coefficient of SMF & $17 \mathrm{ps} / \mathrm{ns} / \mathrm{km}$ \\
Dispersion slope of SMF & $0.075 \mathrm{ps} / \mathrm{nm} / 2 / \mathrm{km}$ \\
Attenuation coefficient of SMF & $0.2 \mathrm{~dB} / \mathrm{km}$ \\
Gain of EDFA1 & $5 \mathrm{~dB}$ \\
Noise figure of EDFA1 & $4 \mathrm{~dB}$ \\
Gain of EDFA2 & $10 \mathrm{~dB}$ \\
Noise figure of EDFA2 & $4 \mathrm{~dB}$ \\
Number of loops & 13 for DB label \\
& 25 for PPM label \\
\hline
\end{tabular}

\section{Result and discussions}

Simulation results and discussions of POLMUX-CSRZ-DQPSK/DB system and POLMUX-CSRZ-DQPSK/PPM system are given as follows.

\subsection{POLMUX-CSRZ-DQPSK/DB system}

ER is the ratio of two optical power levels of a digital signal generated by an optical source. The ER may be given by $E R=P_{1} / P_{0}$, where $P_{1}$ is the optical power level generated when the light source is on, and $\mathrm{P}_{0}$ is the power level generated when the light source is off. For intensity modulation formats, large ER value contributes to good receiver performance, but may be associated with bad receiver performance of phase modulation formats. In OLS system, label and payload transport as combined modulation format, so we should choose an optimized ER to ensure good receiver performance of label and payload.

Q factor as one of important index of network, high Q value means good receiver performance. The Q performance of POLMUX-CSRZ-DQPSK/DB system at different ERs is shown in Figure 2. Different polarization components have different receiver performance. From Figure 2, the Q value of payload components decrease with the increase of ER, to the contrary of payload, the Q value of label increases with the increase of ER. The ER conflict between phase modulation and intensity modulation is always a problem to be solved. To ensure good transmission performance of payload and label, the intersection of label and the worst component of payload is selected, ER of the system is optimized as $2 \mathrm{~dB}$ here.

Figure 3 shows the transmission characteristics of POLMUX-CSRZ-DQPSK/DB system. When the received power ranges from $-14 \mathrm{dBm}$ to $-5 \mathrm{dBm}$, the $\mathrm{Q}$ value of payload components and DB label are proportional to the receiver power. From Figure 3, pol.Y Q-phase component of payload has 
better receiver performance than other components. When BER is $10^{-9}$, the receiver sensitivity of pol.Y Q-phase component is about $-8 \mathrm{dBm}$, the other components are about $-6.386 \mathrm{dBm}$ and the label is about $-5.924 \mathrm{dBm}$. With bit rate of $100 \mathrm{~Gb} / \mathrm{s}$ and transmission distance of $1560 \mathrm{~km}$, the payload and label have good eye diagrams. This means that the proposed system is a potential candidate for the future high bit-rate and long distance OLS network.

The Q performance of DB label and ASK label with same payload at different bit rates is shown in Figure 4. Because high receiver power contribute to the influence of nonlinear effect, the receiver performance in Figure 4 looks not so good. However, comparing DB label with ASK label, we find that when the bit rate of labels are $1.5 \mathrm{~Gb} / \mathrm{s}$ and $2.5 \mathrm{~Gb} / \mathrm{s}$, the Q value of DB label is close to ASK label under the same receiver power. When the bit rate of labels increase to $5 \mathrm{~Gb} / \mathrm{s}$, the Q performance of DB label is greater than ASK label obviously. This is consistent with the theory of bit error rate (BER) of DB in [10], although the concrete values are somewhat different. DB label is more suitable for fast optical label switching systems.

3.2 POLMUX-CSRZ-DQPSK/PPM system

The Q performance of P O L M U X - CSR Z- D Q P SK / P P M system and P O L M U X -CSRZ - DQPSK / ASK system at different ERs is shown in Figure 5. Same as POLMUX-CSRZ-DQPSK/DB system, we should set an ER to ensure good receiver performance of payload and label. From Figure 5, with the increase of ER, the Q value of the payload of PPM decrease more slowly than payload of ASK. The ER value of intersection of POLMUX-CSRZ-DQPSK/PPM system is bigger than that of POLMUX-CSRZ-DQPSK/ASK system. The Q value of PPM label in optimized ER value has about $0.15 \mathrm{~dB}$ gain than the ASK label. For PPM can solve the ER conflict of intensity modulation and phase modulation to a certain extent. In the proposed POLMUX-CSRZ-DQPSK/PPM system, ER is optimized at $0.6 \mathrm{~dB}$ for a high Q value of payload and an acceptable $Q$ value of label.

The BER performance of payload before and after PPM label erased over $3000 \mathrm{~km}$ transmission is shown in Figure 6. For influence of the label, the received power of payload before label erased is $-8.054 \mathrm{dBm}$ when BER is $10^{-9}$, and after label erased, it is $-7.59 \mathrm{dBm}$, the power penalty of label erased is $0.464 \mathrm{dBm}$. It can be seen from the eye diagram that, the system performance is still acceptable in $3000 \mathrm{~km}$ transmission.

In Figure 7, the BER of payload with PPM label and ASK label are demonstrated respectively. The receiver sensitivity of payload with PPM label is about $-8.054 \mathrm{dBm}$, and that of payload with ASK label is $-7 \mathrm{dBm}$ when BER is 10-9. Compared with ASK, the receiver sensitivity of payload of PPM has saved about $1 \mathrm{dBm}$ power consumption. PPM label has greater advantage when a high receiver sensitivity of payload is required.

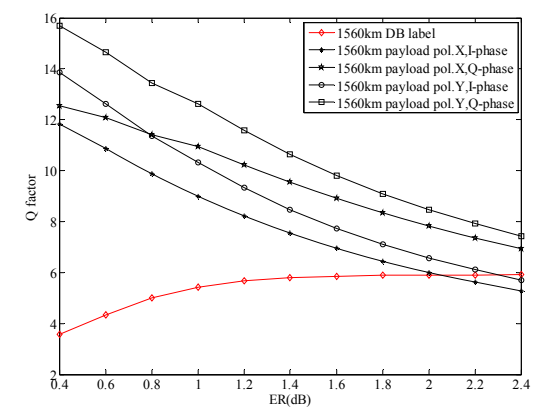

Fig.2 Q performance of POLMUX-CSRZ-DQPSK/DB system at different ERs 


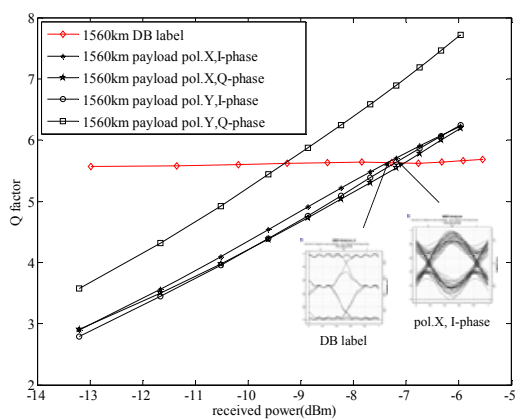

Fig.3 Transmission characteristics of POLMUX-CSRZ- DQPSK/DB system over 1560km

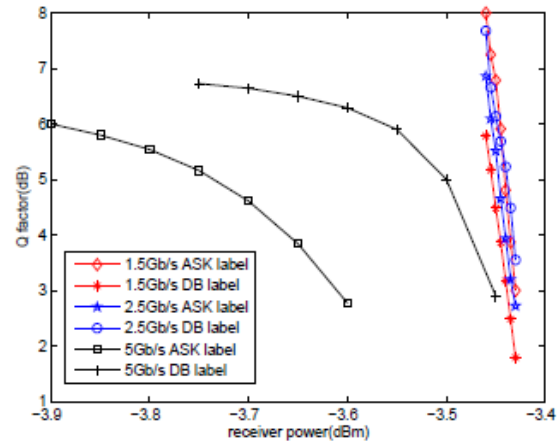

Fig.4 Q performance of DB label and ASK label at different bit rates

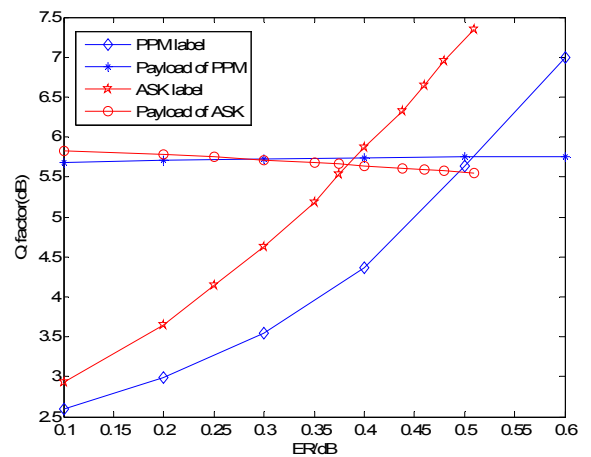

Fig.5 Q performance of POLMUX-CSRZ-DQPSK/PPM system and POLMUX-CSRZDQPSK/ASK system at different ERs

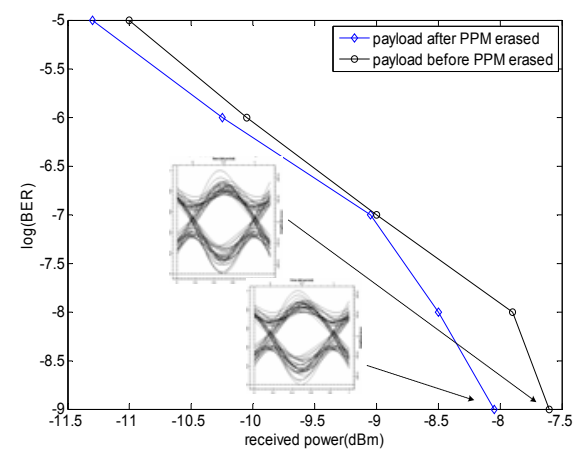

Fig.6 BER performance of payload before and after PPM label erased 


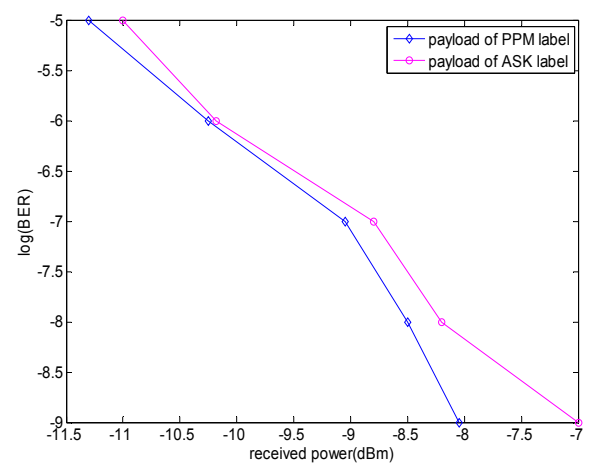

Fig.7 BER performance of payload with PPM label and ASK label

\section{Conclusion}

Two optimized orthogonal OLS schemes based on high bit-rate transport network with rate of $100 \mathrm{~Gb} / \mathrm{s}$ are proposed in this paper. DB and PPM used as labels and combined with POLMUX-CSRZ-DQPSK payload. Comparison between DB label and ASK label, PPM label and ASK label by simulation are given. The maximum transmission distance of POLMUX-CSRZ-DQPSK/DB system and POLMUX-CSRZ-DQPSK/PPM system are $1560 \mathrm{~km}$ and $3000 \mathrm{~km}$, respectively. With the same transmission distance and payload, the receiver performance of DB label is better than ASK label when bit rate is $5 \mathrm{~Gb} / \mathrm{s}$, DB label is more suitable for fast optical label switching systems. Under the same condition, the receiver sensitivity of payload with PPM label increased about $1 \mathrm{dBm}$ than that of payload with ASK label, PPM label has greater advantage when a high receiver sensitivity of payload is required. Two schemes proposed can be potential candidates for the future high bit-rate long distance OLS network.

\section{References}

[1]. Michael Hickey, Charles Barry, Ciro Noronha, and Leonid Kazovsky. Experimental PSK/ASK Transceiver for the STARNET WDM Computer Communication Network. IEEE Photonics Technology Letters. Vol. 5 (1993) No. 5, p. 568-571.

[2]. Michael Hickey and Leonid Kazovsky. The STARNET Coherent WDM Computer Communication Network: Experimental Transceiver Employing a Novel Modulation Format. Journal of Lightwave Technology. Vol.12 (1994) No. 5, p. 876-884.

[3]. Michael Hickey and Leonid Kazovsky, Fellow, IEEE. Combined Frequency and Amplitude Modulation for the STARNET WDM Computer Communication Network. IEEE Photonics Technology Letters. Vol.6 (1994) No. 12, p. 1473-1475.

[4]. HUAWEI, http://www.huawei.com/cn/products/transport-network/index.htm .

[5]. T. He, N. K. Fontaine, R. P. Scott, D. J. Geisler, J. P. Heritage, and S. Yoo. Optical arbitrary waveform generation-based packet generation and all-optical separation for optical-label switching. IEEE Photonics Technology Letters. Vol. 22 (2010) No.10, p 715-717.

[6]. Tafur Monroy, E.J.M.Verdurmen, S.Sulur, A.M.J.Koonen, H.de Waardt, G.D. Khoe, N.Chi, P.V.Holm-Nielsen, J.Zhang, and C.Peucheret. Performance of a SOA-MZI wavelength converter for label swapping using combined FSK/IM modulation format. Optical Fiber Technology. Vol.10 (2004), p. 31-49.

[7]. Kengo Mizote, Hiroki Kishikawa, Nobuo Goto, and Shin-ichiro Yanagiya. Optical label routing processing for BPSK labels using complex-valued neural network. Journal of Lightwave Technology. Vol.31(2013) No. 12, p. 1867-1876. 
[8]. Hongwei Chen, Minghua Chen, and Shizhong Xie. PolSK label over VSB-CSRZ payload scheme in AOLS network. Journal of Lightwave Technology. Vol.25 (2007) No.6, p. $1348-1355$.

[9]. K. Hu, L. Wu, and P. Y. Chiang. A comparative study of $20-\mathrm{gb} / \mathrm{s} \mathrm{NRZ} \mathrm{and} \mathrm{duobinary} \mathrm{signaling}$ using statistical analysis. Transactions on very Large Scale Integration (VLSI) Systems, IEEE. Vol.20 (2012) No.7, p. 1336-1341.

[10]. G. Bosco, A. Carena, V. Curri, R. Gaudino, and P. Poggiolini. Quantum Limit of Direct-Detection Receivers Using Duobinary Transmission. IEEE Photonics Technology Letters. Vol.15 (2003) No.1, p. 102-104.

[11]. I. Ray, M. J. Sibley, and P. J. Mather. Performance analysis of offset pulse-position modulation over an optical channel. Journal of Lightwave Technology. Vol.30 (2012) No.3, p. $325-330$. 